\title{
Study of Large-Signal Stability of an Inverter-based Generator using a Lyapunov Function
}

\author{
Fabio Andrade, Konstantinos Kampouropoulos, Luis \\ Romeral \\ Departament d'Enginyeria Electrica, \\ Universitat Politècnica de Catalunya \\ Terrassa, Spain \\ fabio.andrade@mcia.upc.edu
}

\author{
Juan Carlos Vasquez, Josep M. Guerrero \\ Department of Energy Technology \\ Aalborg University \\ Aalborg, Denmark \\ far@et.aau.dk
}

\begin{abstract}
This document analyses the large-signal stability for an inverter-based generator such as photovoltaic and wind power sources. The objective of this study is to determine the stability region taking into account the electrical and control signal of the generator. The generator uses the concept of the electrostatic machine for the model of the generator. Finally, the applied procedure to find the Lyapunov's function is the Popov method, which not only permits to generate a valid function but also to determine the stability region of the system.
\end{abstract}

Keywords-Microgrid control; Microgrid model; stability of Microgrids

\section{INTRODUCTION}

Generally, the study of the control and the stability of the inverter-based generators is made by the use of a differential equation set that describes the output filter of the inverter and the control loop of the sharing power. This loop contains the equations of the droop curves, the PID controllers and the lowpass filters [4-6]. The model of the generator is analyzed in terms of small-signal in order to evaluate the behavior of its poles and zeros under different variations of the generator's parameters.

The majority of the control strategies that have been used so far are working by imitating the asynchronous machine's behavior and using the control loops to share the active and reactive power [7-10]. In recent studies, authors have tried to find equivalent models between the inverter-based generators and the synchronous machines in order to study their general stability and to improve their operation control [11-13]. Different types of controls, based on the small signal analysis have been proposed on the past to improve the stability of the systems. On [14-16] some studies of the root locus analysis were presented while other authors used non lineal techniques such as the method of Lyapunov [17-18].

The model that is presented in [13] introduces the concept of the electrostatic machine. This concept can be used as a tool to analyze the transferred energy from the DC link to the AC link even in case of large variations of supplied energy, i.e., large variations in DC voltage level.

By the use of this methodology, it is able to access to the motion equations of the generators and to study the stability of the system from the energetic point of view.
This paper is handling with the studying of large-signal stability for an inverter-base generator by means of the Lyapunov function. The objective of the study is to determine the stability region. The concept of the electrostatic machine and the stability analysis by means of the Lyapunov's method have been used for the analysis. The Popov method, which permits to find a valid Lyapunov's function and to determine the stability's region of the system, has been used in this study.

\section{MODEl OF A GENERATOR INTO A MiCROGRID}

A Microgrid system with DC-AC inverted-based generators and power sharing loads has been considered. Figure 1 depicts a renewable generator that is being fed by a DC link. The prime energy of this generator, which can be solar or wind, is transferred as AC power through an inverter and an intelligent control system. The control system uses a PLL block and inner control loops of current and voltage to ensure the respective reference signals. The output current and voltage reference signals are configured by an outer control loop of sharing power which uses the basic idea of the synchronous generator concept to control the sharing power by using droop curves (" $P$ vs. f" and "Q vs. V").

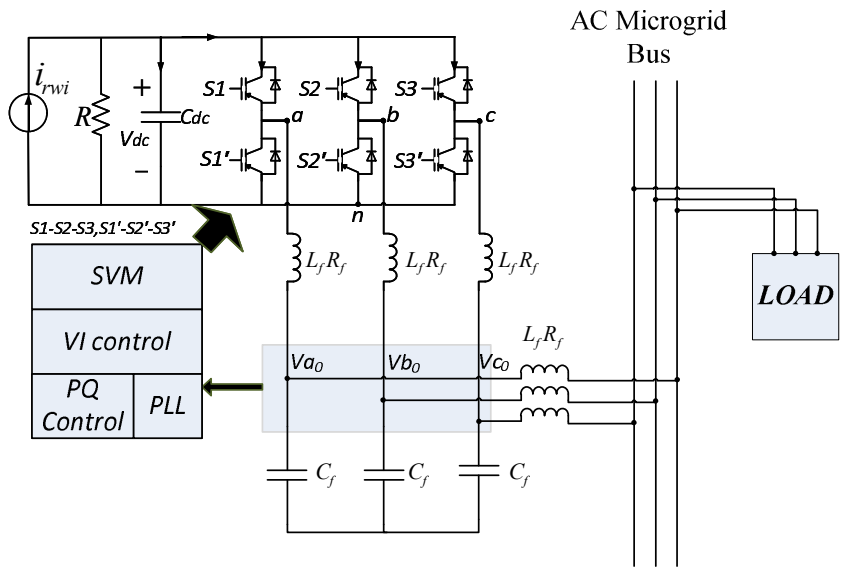

Fig. 1. A renewable inverter-based generator connected to a Microgrid System 


\section{A. Electrostatic machine concept applied to generator model}

The generator can be modeled by using the electrostatic machine concept as it was proposed in [13]. The electrical equivalent circuit of the generator is presented in Fig. 2.
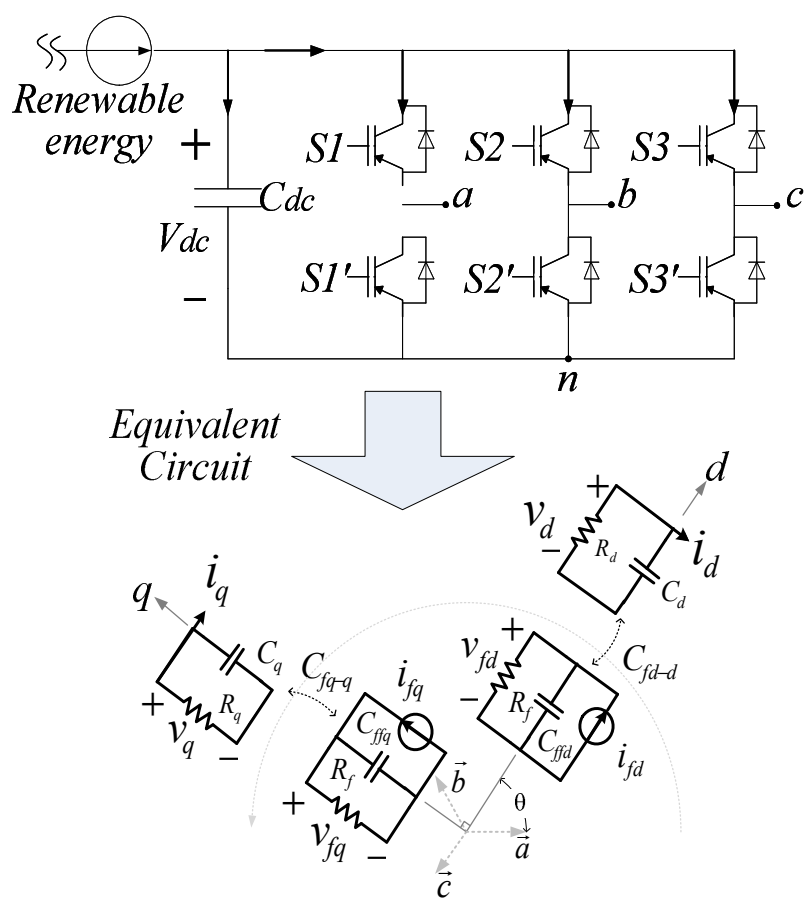

Fig. 2. Equivalent circuit of the Microgrid is presented.

The swing equation of the magnetic synchronous machine (1) can be also applied for the electrostatic machine concept.

$$
\begin{aligned}
& \frac{d \Delta \omega_{r}}{d t}=\frac{1}{2 H}\left(P_{m}-P_{e}-K_{D} \Delta \omega_{r}\right) \\
& \frac{d \delta}{d t}=\omega_{0} \Delta \omega_{r}
\end{aligned}
$$

The above equation is normalized in term of per unit inertia constant $H$, defined as the "kinetic" energy in watt-seconds at rated speed divided by the rate power value (VAbase). The $\mathrm{H}$ value is calculated for the electrostatic machine by making equal the stored energy and the kinetic energy

$$
\frac{1}{2} C V_{d c}^{2}=\frac{1}{2} J \omega_{r}^{2} \rightarrow H=\frac{C V_{d c}^{2}}{2 V A_{b a s e}}
$$

The damping power is proportional to the frequency deviation $D i$ and is closely associated with the "P vs. Q" droop curve. The inertia constant of the machine $\left(M_{i}\right)$ is defined as $H_{i} / \pi f_{0}$. By the use of the swing equation, it is possible to analyze the actual energy at the virtual rotor, i.e., the available $\mathrm{DC}$ energy, the control that is required to inject the energy to the AC link and finally the system's behavior under different disturbances. The steady state analysis of the electrostatic machine permits to model its equivalent circuit, calculating the values of voltage, current and impedance and determining the power flows from the generator to the Microgrid. Figure 3 depicts the equivalent circuit of the generator.

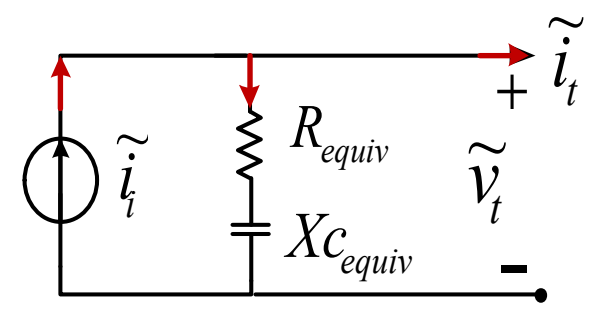

Fig. 3. Equivalent electric circuit of an inverter-based generator.

The voltage and current of the machine's stator can be expressed as phasors $v_{t}$ and $i_{t}$ respectively in the standard $D Q$ Cartesian plane, where $v_{t}=v_{d}+j v_{q}$ and $i_{t}=i_{d}+j i_{q}$.

The input current $\left(i_{i}\right)$ equation can be calculated in function of the rotor's voltages and its capacitive impedances:

$$
\widetilde{i_{i}}=\frac{1}{X c_{f q-q}} V_{f q}-j \frac{1}{X c_{f d-d}} V_{f d}
$$

The equivalent reactance's and voltage's equations are described on detail on (4), where their values depend on the inverter's parameters.

$$
\begin{aligned}
& X c_{f d-d}=X c_{f q-q}=X c_{d c}=\left(\omega C_{d c}\right)^{-1} \\
& V_{f q}=\frac{V_{d c}}{2} d_{q} \quad V_{f d}=\frac{V_{d c}}{2} d_{d}
\end{aligned}
$$

Where $d$ is the average duty cycle of the inverter. Finally, the equivalent resistance and reactance can be expressed as follows, combining the above equations:

$$
\begin{aligned}
& X c_{\text {equiv }}=X c_{d c}\left(\frac{d_{q}^{2}-d_{d}^{2}}{d_{q}^{2}+d_{d}^{2}}\right) \\
& R_{\text {equiv }}=X c_{d c}\left(\frac{2 d_{q} d_{d}}{d_{q}^{2}+d_{d}^{2}}\right)
\end{aligned}
$$

\section{B. Model of the generators}

Every generator that includes a power electronic interface like the one presented in Fig. 1, has an equation that describes its movement and can be used to analyze its stability range. In order to formulate this equation, the equivalent electric circuit is used (Fig. 4), determining the power flows during the steady state. The admittances of each branch in this circuit can be expressed as follows: 


$$
\begin{aligned}
& Y_{e q}=\left(R_{e q}-j X_{e q}\right)^{-1} ; Y_{f}=\left(R_{f}+j X_{f}\right)^{-1} ; Y_{l}=\left(R_{l}-j X_{l}\right)^{-1} \\
& Y_{c}=\left(-j X_{c}\right)^{-1} ; \vec{i}_{\mu G}=Y_{l} \cdot \vec{v}_{\mu G}
\end{aligned}
$$

The matrix of the minimum impedances of the circuit can be calculated by simplifying the equivalent circuit. This matrix is presented in (6).

$$
\left[\begin{array}{c}
\vec{i}_{i} \\
\vec{i}_{\mu G}
\end{array}\right]=\underbrace{\left[\begin{array}{cc}
Y_{A} & Y_{B} \\
Y_{B} & Y_{D}
\end{array}\right]}_{Y_{\text {bus }}}\left[\begin{array}{l}
\vec{v}_{1} \\
\vec{v}_{2}
\end{array}\right] \rightarrow Z_{\text {bus }}=Y_{\text {bus }}^{-1}
$$

The expression to calculate the reactive power of the generator can be expressed as:

$$
\begin{aligned}
& P_{e}=\operatorname{Re}\left\lfloor\vec{v}_{2} \cdot \vec{i}_{\mu G}^{*}\right\rfloor \\
& P_{e}=\left|I_{i}\right|^{2} R_{A}+F_{12} \sin \left(\delta_{1}-\delta_{2}\right)+G_{12} \cos \left(\delta_{1}-\delta_{2}\right)
\end{aligned}
$$

Where

$$
\begin{array}{lr}
Z_{m}=R_{m}+j X_{m}=\left|Z_{m}\right| \angle \theta_{Z_{m}} ; & \vec{I}_{i}=\left|I_{i}\right| \angle \delta_{i} ; \quad \vec{I}_{\mu G}=\left|I_{\mu G}\right| \angle \delta_{\mu G} \\
F_{12}=\left|I_{i}\right|\left|I_{\mu G}\right|\left|Z_{B}\right| \cos \left(\theta_{Z_{B}}\right) & G_{12}=\left|I_{i}\right|\left|I_{\mu G}\right|\left|Z_{B}\right| \sin \left(\theta_{Z_{B}}\right) \\
m=A, B, C, D &
\end{array}
$$

Finally, the generator's model can be described as follows, by using the equation (1).

$$
\begin{aligned}
& M \frac{d^{2} \delta_{1}}{d t^{2}}+D \frac{d \delta_{1}}{d t}=P-P_{e} \\
& P=P_{m}-\left|I_{i}\right|^{2} R_{A} \\
& P_{e}=F_{12} \sin \left(\delta_{1}-\delta_{2}\right)+G_{12} \cos \left(\delta_{1}-\delta_{2}\right)
\end{aligned}
$$

\section{Model of the generators}

The parameters that describe the inertial and damping values of the generator are expressed as $\mathrm{M}$ and $\mathrm{D}$ respectively in the following equations.

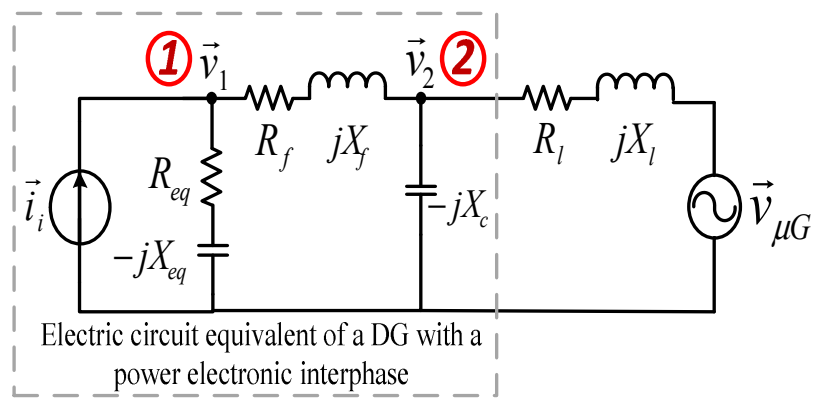

Fig. 5. Steady state equivalent circuit.
The D constant indicates a frequency's deviation in function of the delivery power by each generator. In this type of generators that are based on DC/AC interfaces, the frequency's deviation occurs because of the droop curves that are used on the droop curves in the shared power's control. The block diagram of Fig. 5 presents the relation of the reference's frequency in function of the power that is shared to the Microgrid. The relations that express the power and the frequency deviation $(\Delta \omega)$ are presented in (9).

$$
\begin{aligned}
& \Delta \omega=\frac{\omega_{f}}{s+\omega_{f}}\left(-K_{p} \Delta P\right) \\
& \Delta P=\left(-\frac{1}{\omega_{f} K_{p}} \frac{d \omega}{d t}-\frac{1}{K_{p}}\right) \Delta \omega
\end{aligned}
$$

Combining the movement's equation that is described in (6) and the equation of the power control, it is able to calculate the Mi and Di parameters as follows:

$$
M=\frac{2 H K_{p} \omega_{f}+1}{K_{p} \omega_{f}}, D=\frac{1}{K_{p}}
$$

The parameters $F$ and $G$ make reference to the existed relation between the impedance of the line that connects the grid, the output filters of the generator and the load impedance.

The power $P_{m}$ can be determined with the maximum value of the available power on the DC bus and the maximum values of the signals $d_{d}$ and $d_{q}$ of the control.

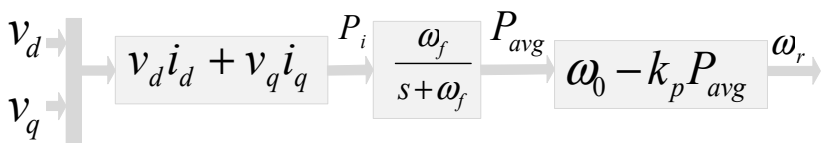

$$
\begin{aligned}
& i_{d} \Rightarrow v_{q} i_{d}-v_{d} i_{q} Q_{i} \frac{\omega_{f}}{s+\omega_{f}} Q_{a v g} v_{0}-k_{v} Q_{a v g} v_{r e f} \\
& i_{q} \Rightarrow \text { Active and Reactive Low-pass } \\
& \text { Power Filter }
\end{aligned}
$$

Fig. 4. Equivalent circuit of the Microgrid is presented.

\section{Equilibrium points of the System}

The equilibrium points can be found by expressing the derivatives of the system as equal to zero. In this way, the power of the input of the DC link of the generator is equal to the AC power that is supplied to the grid by the generator $\left(P_{I}=P_{e l}\right)$. The obtained equation expresses relation of the angles of each generator. The solution of this set of equations in an interval of $\pi$ to $-\pi$ is: 


$$
\begin{aligned}
& P_{e}=\sqrt{F_{12}^{2}+G_{12}^{2}} \sin \left(\delta_{1}-\delta_{2}+\tan ^{-1}\left(F_{12} / G_{12}\right)\right) \\
& \text { (i) } \delta_{1}^{s}-\delta_{2}^{s}=\sin \left(P_{e} / \sqrt{F_{12}^{2}+G_{12}^{2}}\right)-\tan ^{-1}\left(F_{12} / G_{12}\right) \\
& \text { (ii) } \delta_{1}^{u}-\delta_{2}^{u}=\pi-\sin \left(\frac{P_{e}}{\sqrt{F_{12}^{2}+G_{12}^{2}}}\right)-\tan ^{-1}\left(F_{12} / G_{12}\right)
\end{aligned}
$$

It is easily verified by linearization of (8) that $\left(\delta_{1}^{s}-\delta_{2}^{s}\right)$ is the stable point and $\left(\delta_{1}^{u}-\delta_{2}^{u}\right)$ is the unstable equilibrium point.

\section{LYAPUNOV STABILITY OF A GENERATOR INTO THE MICROGRID}

\section{A. Transfer of the stable equilibrium point to the origin}

For Lyapunov stability issues, the mathematical model of the Microgrid is transferred from the stable equilibrium point to the origin point, by using the transformation $y=\delta-\delta^{s}$. The state variables representation can be formulated as follows:

$$
\begin{aligned}
& y_{2}=\delta_{12}-\delta_{12}^{s} \quad y_{1}=\omega_{1} \\
& \left\{\begin{array}{l}
\frac{d y_{1}}{d t}=-\frac{D}{M} y_{1}-\frac{1}{M} f\left(y_{2}\right)-\frac{1}{M} g\left(y_{2}\right) \\
\frac{d y_{2}}{d t}=y_{1}
\end{array}\right. \\
& f\left(y_{2}\right)=F_{12}\left[\sin \left(y_{2}+\delta_{12}^{s}\right)-\sin \left(\delta_{12}^{s}\right)\right] \\
& g\left(y_{2}\right)=G_{12}\left[\cos \left(y_{2}+\delta_{12}^{s}\right)-\cos \left(\delta_{12}^{s}\right)\right]
\end{aligned}
$$

The model can be expressed in matrix formulation as:

$$
\begin{aligned}
& \dot{Y}=A Y-B_{1} f\left(y_{2}\right)-B_{2} g\left(y_{2}\right) \\
& A=\left[\begin{array}{cc}
-\lambda & 0 \\
1 & 0
\end{array}\right] B_{1}=\left[\begin{array}{c}
\frac{1}{M_{1}} \\
0
\end{array}\right] B_{2}=\left[\begin{array}{c}
\frac{1}{M_{1}} \\
0
\end{array}\right] \\
& f\left(y_{2}\right)=F_{12}\left[\sin \left(y_{2}+\delta_{12}^{s}\right)-\sin \left(\delta_{12}^{s}\right)\right] \\
& g\left(y_{2}\right)=G_{12}\left[\cos \left(y_{2}+\delta_{12}^{s}\right)-\cos \left(\delta_{12}^{s}\right)\right]
\end{aligned}
$$

Where $\lambda_{i}=\frac{D_{i}}{M_{i}}$

\section{B. Lyapunov Stability}

Considering that the network between the generators has an inductive behavior, (i.e. $\theta_{Z_{B}}=\pi / 2$ ) then the value of the parameter $G_{12}$ is equal to zero. This consideration has as a result that the system in (13) can be expressed as it is presented in Fig. 6. The transfer function of the linear part is given by:

$$
W(s)=C(s I-A)^{-1} B_{1}
$$

So far, the generator has been modeled and represented as a block diagram (presented in Fig. 6) which consists of a linear transfer function and a nonlinear function in the feedback path.

If the nonlinear function lies in the first and the third quadrant of Fig. 7, then it is possible to construct the Lyapunov's function by applying a systematic procedure.

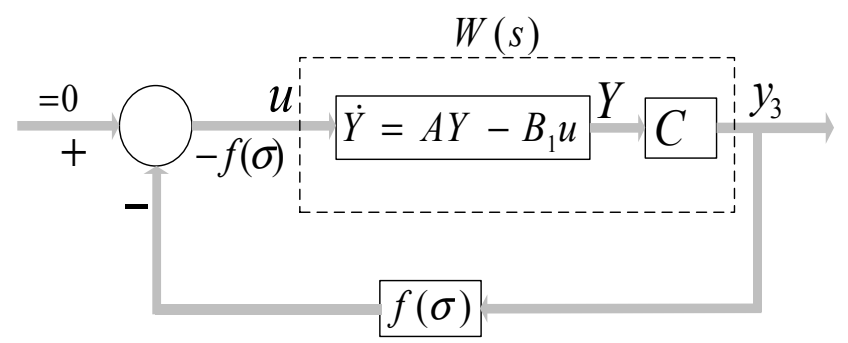

Fig. 6. Block diagram representation of a multi-power systems model.

One of these systematic procedures that are commonly used is the Popov's method. This method allows to generate the Lyapunov's function and to construct a region of attraction for the equilibrium point.

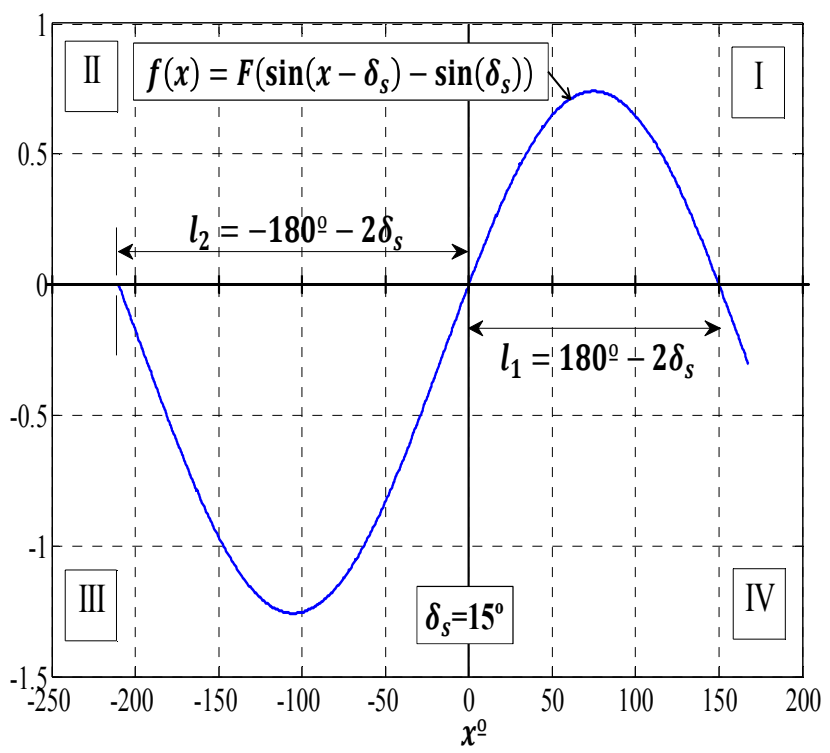

Fig. 7. The nonlinearity of function $\mathrm{f}(\mathrm{x})$

In order to detect the boundaries of the stability region of the generator, a conservative system without energy loss is considered as the most critical possible scenario. In this case, the stored energy in the DC bus is transferred to the AC bus. As it is presented on (15), the DC energy is considered as kinetic, while the AC energy is considered as potential. The equations of these energies, potential and kinetic, are combined to construct the Lyapunov's function by applying the Popov's method. 


$$
\begin{aligned}
& V\left(y_{1}, y_{2}\right)=\frac{1}{2} M y_{1}^{2}+\int_{0}^{y_{2}} f(u) d u \\
& V\left(y_{1}, y_{2}\right)=\frac{1}{2} M y_{1}^{2}+F_{12} \sin \left(y_{2}+\delta_{12}^{s}\right)-F_{12} \sin \left(\delta_{12}^{s}\right)
\end{aligned}
$$

This function fulfills the following properties:

- $V(0)=0$

- $\quad V\left(y_{1}, y_{2}\right)>0$ for all $\mathrm{y}_{1}, \mathrm{y}_{2}$

- $\frac{d}{d t} V\left(y_{1}, y_{2}\right) \leq 0$ along all trajectories of the system.

Then the point $\left(y_{1}, y_{2}\right)=0$ is locally stable.

According to the initial condition of the function $\mathrm{f}(\mathrm{x})$, it should be lying on the first and third quadrant. This function is used to detect the attraction area, evaluating the angles 11 and 12 at the Lyapunov's function and finding which one takes the minimum values of the function between those angles.

From the above calculations, it can be observed that every value of angle that is evaluated by the Lyapunov's function and results to be higher than the limit (11 or 12), will be outside of the attraction area and will create an unstable condition for the system.

In the next step, the analysis of the generator that is connected to a Microgrid will be presented, finding the stability region of the system.

\section{SimULATIONS}

Simulations based on Hardware in the loop (HIL), by using a dSPACE ds1006, MATLAB/Simulink and dSPACE Control Desk, are performed to evaluate the proposed method for study the large-signal stability. In the case of study, an inverter-based generator is connected to a Microgrid working in island-mode.

Electrical setup and control systems parameter are listed in table I.

\begin{tabular}{|c|c|c|}
\hline Type & Parameters & Value \\
\hline \multirow{7}{*}{ 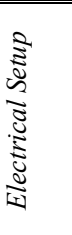 } & $\overline{D C}$ D voltage $\left(V_{d c}\right)$ & (650V \\
\hline & $A C$ voltage $\left(V_{\mu G}\right)$ & $311 \mathrm{~V}$ \\
\hline & $A C$ frequency $(F)$ & $50 \mathrm{~Hz}$ \\
\hline & DC capacitance $\left(C_{d c}\right)$ & $5000 \mu \mathrm{F}$ \\
\hline & $A C$ filter capacitance $\left(L_{f}\right)$ & $25 \mu \mathrm{F}$ \\
\hline & AC filter impedance $\left(L_{f}\right)$ & $1.8 \mathrm{mH}$ \\
\hline & AC output impedance $\left(L_{f}\right)$ & $1.8 \mathrm{mH}$ \\
\hline \multirow{4}{*}{ 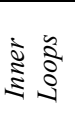 } & Active power droop control $\left(K_{P}\right)$ & $0.00001 \mathrm{~W} / \mathrm{rad}$ \\
\hline & Reactive power droop control $\left(K_{Q}\right)$ & $0.0001 \mathrm{VAr} / \mathrm{rad}$ \\
\hline & Virtual resistance $\left(R_{v}\right)$ & $0.1 \Omega$ \\
\hline & Virtual inductance $\left(L_{v}\right)$ & $4 \mathrm{mH}$ \\
\hline
\end{tabular}

TABLE I. ELECTRICAL SETUP AND CONTROL SyStem Parameters

Analyzing the data that were obtained from the HIL systems, the equivalent circuit of the stable state was calculated. The equivalent circuit of the generator for the stable state is depicted in Fig. 8. The equilibrium points of the generator are:

- Stable equilibrium point: $\delta_{s}=1.5162$

- Unstable equilibrium point: $\delta_{u}=1.6254$

To analyze the model by means of Lyapunov's methodology is necessary to locate the model on its stable equilibrium point. The model on its steady state can be expressed as follows by using the electrostatic concept.

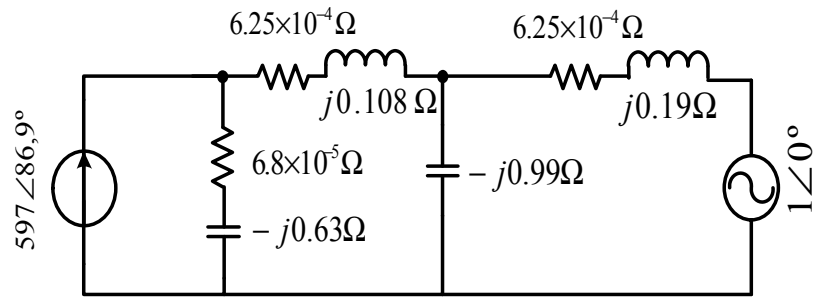

Fig. 8. Equivalent circuit of the steady state of the generator

$$
\left\{\begin{array}{l}
\frac{d y_{1}}{d t}=y_{2} \\
\frac{d y_{2}}{d t}=-\frac{1}{5.86} f\left(y_{1}\right)
\end{array}\right.
$$

$$
f\left(y_{1}\right)=8,36\left(\sin \left(y_{1}+1.5162\right)-\sin (1.5162)\right)
$$

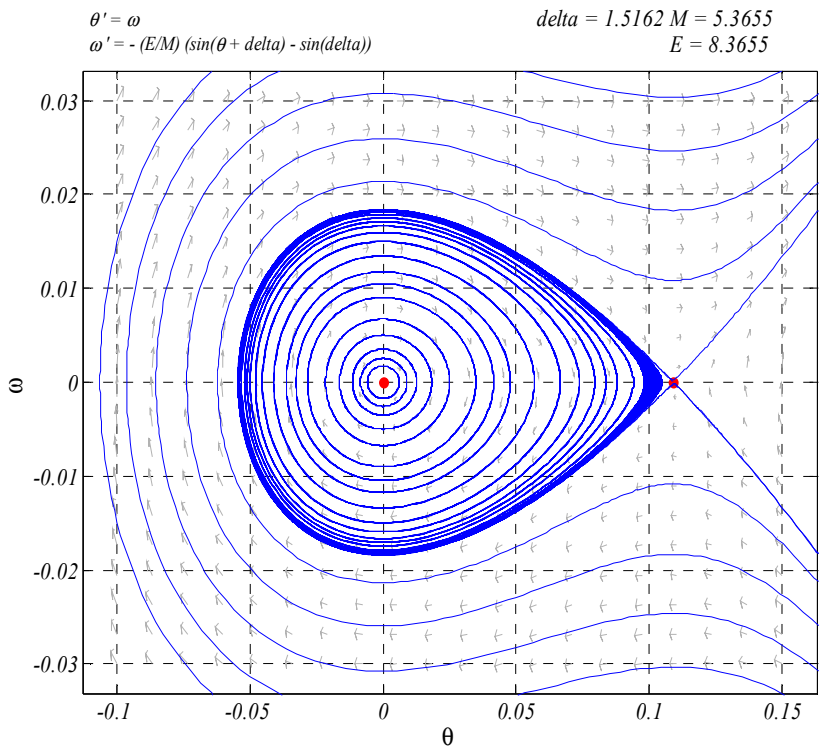

Fig. 9. Portrait plane of the generator. 


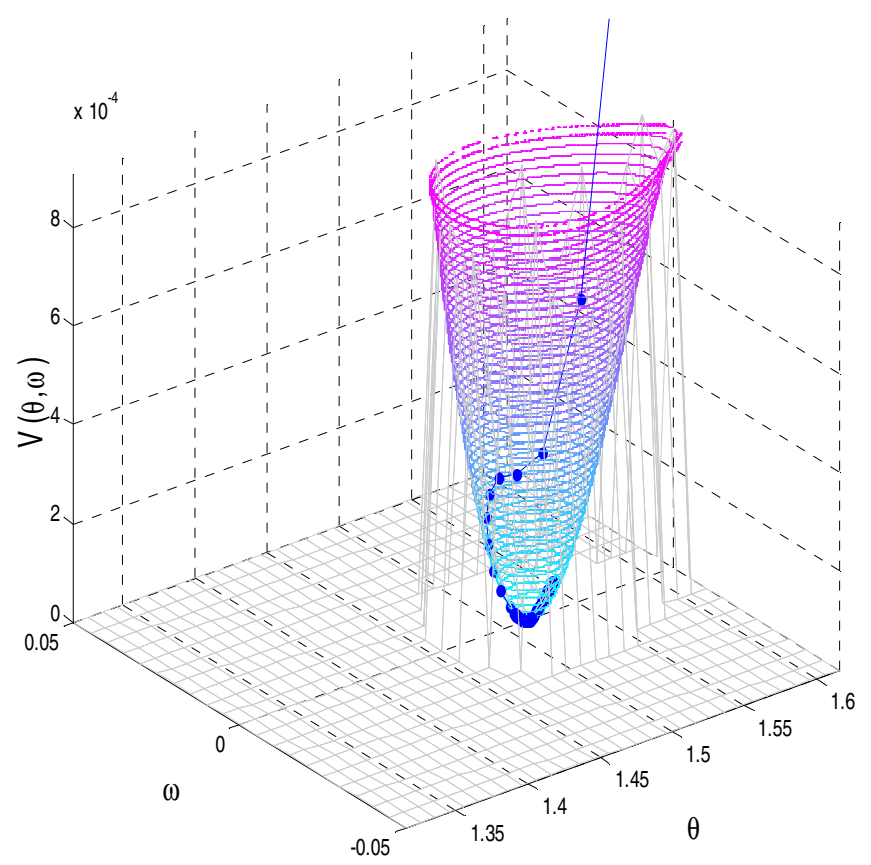

(a)

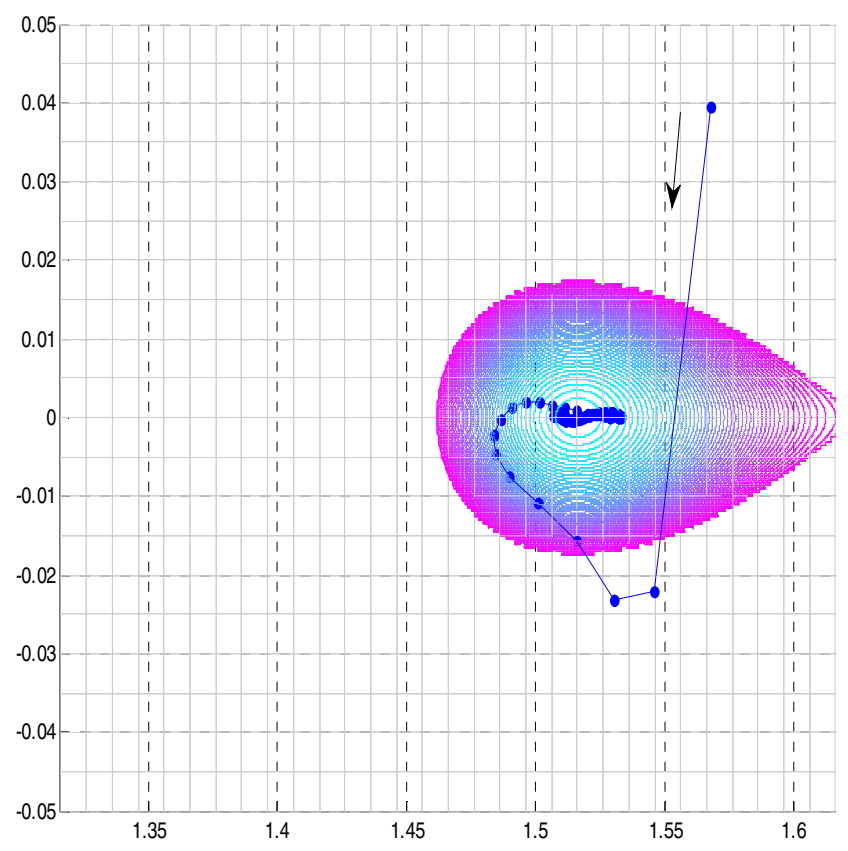

(b)

Fig. 10 Analysis of Lyapunov to the data of frequency and power angle.
The D parameter depends on the control unit. The case where the D is equal to zero is very interesting, because it permits to generate the maximum region of stability on the system in function of the available energy.

Using the Popov's method, it is able to determine the stability of the equilibrium point as well as the region in which it is possible to recuperate the system (return to a stable condition from a disturbance condition).

Figure 10 presents the Lyapunov's function of the generation system, in which it can be observed the existence of a region with a conical shape. In this region, whichever disturbance that doesn't cause an overpass of the frequency's and angles boundaries can be recuperated, returning the system to the equilibrium point. It can be observed that the conical region has as superior limit the value $(V(\phi, \omega)=9,02 e-4)$ which was determined by the restriction of $f(y)$ function and defines the limits of $l_{1}$ and $l_{2}$.

The blue curve presents the data of the frequency and angle variables during the initial moment of the simulation and till the reach of the steady state. The system starts outside of the attraction region but as it enters, it tends towards the equilibrium point. Whichever disturbance that forces the system to take values of $\omega$ and $\theta$ outside of this space has as a result the driving of the system to an unstable condition without the possibility of recuperation.

\section{CONCLUSIONS}

This article contributes to the study and control of the generators with $\mathrm{DC} / \mathrm{AC}$ power interfaces, using a model that is based on the electrostatics' machine concept. Also, on this work, the stability of the system is analyzed by the Lyapunov's theory. To find the Lyapunov's function, the Popov's method has been used, permitting also to calculate the attraction region of the system and its equilibrium point.

\section{ACKNOWLEDGMENT}

The research that leads to these results has received funding from the People program (Marie Curie Actions) of the Seventh Framework Programme of the European Union (FP7/20072013) under grant agreement no. 600388 of REA.

\section{REFERENCES}

[1] J. Rocabert, et al., "Intelligent Connection Agent for Three-Phase GridConnected Microgrids," Power Electronics, IEEE Transactions on, vol. 26, pp. 2993-3005, 2011.

[2] C. Zhe, et al., "A Review of the State of the Art of Power Electronics for Wind Turbines," Power Electronics, IEEE Transactions on, vol. 24, pp. 1859-1875, 2009.

[3] J. M. Guerrero, et al., "Distributed Generation: Toward a New Energy Paradigm," Industrial Electronics Magazine, IEEE, vol. 4, pp. 52-64, 2010.

[4] J. C. Vasquez, et al., "Modeling, Analysis, and Design of StationaryReference-Frame Droop-Controlled Parallel Three-Phase Voltage Source Inverters," Industrial Electronics, IEEE Transactions on, vol. 60, pp. 1271-1280, 2013.

[5] H. Jinwei and L. Yun Wei, "Analysis, Design, and Implementation of Virtual Impedance for Power Electronics Interfaced Distributed 
Generation," Industry Applications, IEEE Transactions on, vol. 47, pp. 2525-2538, 2011.

[6] Y. Wei, et al., "Design and Analysis of the Droop Control Method for Parallel Inverters Considering the Impact of the Complex Impedance on the Power Sharing," Industrial Electronics, IEEE Transactions on, vol. 58 , pp. 576-588, 2011.

[7] J. C. Vasquez, et al., "Adaptive Droop Control Applied to VoltageSource Inverters Operating in Grid-Connected and Islanded Modes," Industrial Electronics, IEEE Transactions on, vol. 56, pp. 4088, 2009.

[8] T. L. Vandoorn, et al., "Active Load Control in Islanded Microgrids Based on the Grid Voltage," Smart Grid, IEEE Transactions on, vol. 2, pp. 139-151, 2011.

[9] K. Jaehong, et al., "Mode Adaptive Droop Control With Virtual Output Impedances for an Inverter-Based Flexible AC Microgrid," Power Electronics, IEEE Transactions on, vol. 26, pp. 689-701, 2011.

[10] J. M. Guerrero, et al., "Hierarchical Control of Droop-Controlled AC and DC Microgrids - A General Approach toward Standardization," Industrial Electronics, IEEE Transactions on, vol. 58, pp. 158-172, 2011.

[11] Z. Qing-Chang and G. Weiss, "Synchronverters: Inverters That Mimic Synchronous Generators," Industrial Electronics, IEEE Transactions on, vol. 58, pp. 1259-1267, 2011.
[12] T. L. Vandoorn, et al., "Analogy between Conventional Grid Control and Islanded Microgrid Control Based on a Global DC-Link Voltage Droop," Power Delivery, IEEE Transactions on, vol. 27, pp. 1405, 2012.

[13] F. Andrade, et al., "New Model of a Converter-based Generator Using Electrostatic Synchronous Machine Concept," Energy conversion, IEEE Transactions on, 2014.

[14] S. M. Ashabani and Y. A. I. Mohamed, "A Flexible Control Strategy for Grid-Connected and Islanded Microgrids With Enhanced Stability Using Nonlinear Microgrid Stabilizer," Smart Grid, IEEE Transactions on, vol. 3, pp. 1291-1301, 2012.

[15] R. Majumder, et al., "Improvement of Stability and Load Sharing in an Autonomous Microgrid Using Supplementary Droop Control Loop," Power Systems, IEEE Transactions on, vol. 25, pp. 796-808, 2010.

[16] C. N. Rowe, et al., "Arctan Power-Frequency Droop for Improved Microgrid Stability," Power Electronics, IEEE Transactions on, vol. 28, pp. 3747-3759, 2013.

[17] S. V. Iyer, et al., "A Generalized Computational Method to Determine Stability of a Multi-inverter Microgrid," Power Electronics, IEEE Transactions on, vol. 25, pp. 2420-2432, 2010.

[18] S. Dasgupta, et al., "Lyapunov Function-Based Current Controller to Control Active and Reactive Power Flow From a Renewable Energy Source to a Generalized Three-Phase Microgrid System," Industrial Electronics, IEEE Transactions on, vol. 60, pp. 799-813, 2013 